\title{
Initial experience of temperature-controlled irrigated radiofrequency ablation for ischaemic cardiomyopathy ventricular tachycardia ablation
}

\author{
Jaffar Al-Sheikhli ${ }^{1} \cdot$ Ian Patchett ${ }^{1} \cdot$ Ven Gee Lim $^{1}$ Leeann Marshall ${ }^{1} \cdot$ Will Foster $^{1} \cdot$ Michael KuehI $^{1,2}$. \\ Shamil Yusuf ${ }^{1} \cdot$ Sandeep Panikker ${ }^{1} \cdot$ Kiran Patel $^{1,2} \cdot$ Faizel Osman $^{1,2} \cdot$ Prithwish Banerjee $^{1,2} \cdot$ Nicolas Lellouche $^{3}$. \\ Tarvinder Dhanjal ${ }^{1,2}$ (1)
}

Received: 31 December 2021 / Accepted: 10 February 2022 / Published online: 22 February 2022

(c) The Author(s) 2022

\begin{abstract}
Background The DiamondTemp ablation (DTA) catheter system delivers high power, open-irrigated, temperature-controlled radiofrequency (RF) ablation. This novel ablation system has not been previously used for ventricular tachycardia (VT) ablation.

Objective Feasibility of using the DTA catheter system for VT ablation in ischaemic cardiomyopathy (ICM) patients. Method Ten ICM patients with optimal anti-arrhythmic drug therapy and implantable cardiac defibrillators (ICD) were recruited. VT inducibility testing was performed at the end of the procedure. ICD data for device detected VT episodes and device treated VT episodes were collected for 6-months pre- and post-ablation.

Results Substrate analysis demonstrated reductions in the borderzone area of $4.4 \mathrm{~cm}^{2}(p=0.026)$ and late potential area of $3.5 \mathrm{~cm}^{2}(p=0.0449)$ post-ablation, with reductions in the mean bipolar and unipolar voltages of the ablation target areas $(0.14 \mathrm{mV}(p=0.0007) ; 0.59 \mathrm{mV}(p=0.0072)$ respectively). Complete procedural success was achieved in 9 procedures. Postablation VT inducibility testing was not performed in 1 procedure due to a steam pop complication resulting in pericardial tamponade requiring drainage. Mean follow-up of $214 \pm 33$ days revealed an 88\% reduction in total VT episodes $(n=266$ median 16 [IQR 3-57] to $n=33$ median $0 ; p=0.0164)$ and 77\% reduction in ICD therapies $(n=128$ median 5 [IQR 2-15] to $n=30$ median $0 ; p=0.0181$ ).

Conclusion The DTA system resulted in adequate lesion characteristics with effective substrate modification, acute procedural success and improved outcomes at intermediate-term follow-up. Randomised controlled trials are required to compare the performance of the DTA system against conventional ablation catheters.
\end{abstract}

Keywords Ventricular tachycardia $\cdot$ Ischaemic cardiomyopathy $\cdot$ Temperature-controlled irrigated radiofrequency ablation · Feasibility

\section{Introduction}

Jaffar Al-Sheikhli and Ian Patchett are joint first authors

Tarvinder Dhanjal

tarv.dhanjal@uhcw.nhs.uk

1 Department of Cardiology, University Hospital Coventry \& Warwickshire NHS Trust, Coventry, UK

2 University of Warwick, Coventry, UK

3 Avenue du Marechal de Lattre de Tassigny, Hopital Henri Mondor Albert Chenevier, Creteil, Inserm U955, 94000 Paris, France
Current therapeutic approaches for ventricular tachycardia (VT) including invasive catheter ablation (CA) and antiarrhythmic drug (AAD) therapy lack long-term efficacy and are associated with significant side effects [1]. The delivery of transmural and durable lesion creation remains challenging when using traditional focal irrigated radiofrequency $(\mathrm{RF})$ ablation catheters. Treatment failure with CA can be due to the inability to deliver adequate ablative energy across ventricular myocardium as long-term outcome following $\mathrm{CA}$ remains variable, with high rates 
of VT recurrence [1-3]. Thus, there is an urgent need to develop novel CA technologies.

Traditional non-irrigated ablation catheters can have deleterious effects because of overheating, such as char and thrombus formation which is associated with an increased risk of thromboembolic complications [4, 5]. This prompted the development of external irrigation catheters which have reduced the frequency of complications associated with overheating, but at the cost of reduced acuity of thermal feedback from the catheter tip, resulting in challenges with titrating energy during ablation [6]. As a result, traditional irrigated catheters are operated in a 'power control mode', in order to deliver RF energy. To address these limitations, the DiamondTemp ablation (DTA) catheter (Medtronic, Inc., Minneapolis, MN) was designed to re-establish accurate measurement of tissue temperature. This is accomplished via 6 externally located thermocouples and a chemical vapour deposit (CVD) diamond network to shunt heat from the catheter tip housing 6 saline irrigation ports (Fig. 1a). The CVD network allows thermal energy transfer that is 200-400 times faster than achieved with other catheter systems [7]. The catheter delivers high power RF energy up to $50 \mathrm{~W}$ in a 'temperature control mode' with real-time power modulation where power delivery is based on the highest thermocouple temperature reading every $20 \mathrm{~ms}$ (Fig. 1b).

Preclinical data from computational modelling, thermochromic gel and porcine thigh preparation experiments have guided temperature controlled-ablation parameters [8]. In this study, we aim to demonstrate the feasibility of using the DTA catheter system for VT ablation in patients with ischaemic cardiomyopathy (ICM).

\section{Methods}

This prospective, single-centre study recruited patients who underwent CA for VT with ICM between January and April 2021 at the University Hospital Coventry \& Warwickshire (UHCW) NHS Trust.

\subsection{Study population}

Adult patients ( $\geq 18$ years) with an implantable cardiac defibrillator (ICD) and clinical indications for VT ablation including symptomatic VT despite optimised medical therapy, three or more episodes of VT within $24 \mathrm{~h}$, at least 3 episodes of VT requiring anti-tachycardia pacing (ATP), or at least one appropriate ICD shock were included. All patients provided written consent prior to the procedure. Approval for the study was provided by our Local Audit and Research Department. The study applied the principles of the Declaration of Helsinki.

\subsection{Study protocol}

All cases were first time procedures carried out according to the UHCW VT ablation workflow as previously described, and all AADs including amiodarone were discontinued for at least 5 days prior to ablation if the stability of arrhythmia (a)

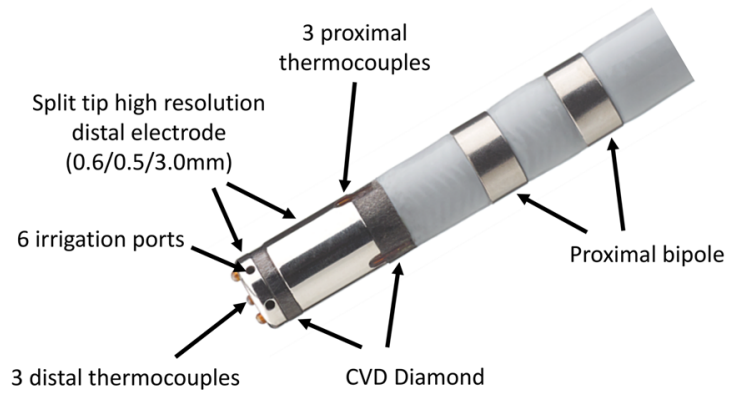

(b)
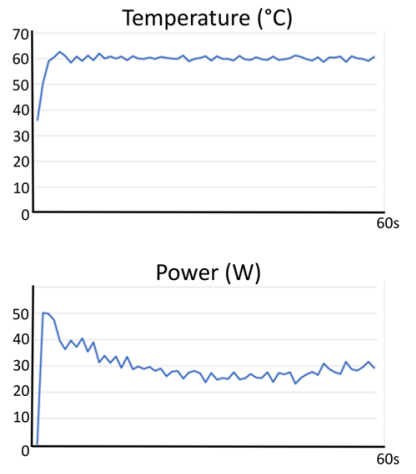

Impedance $(\Omega)$

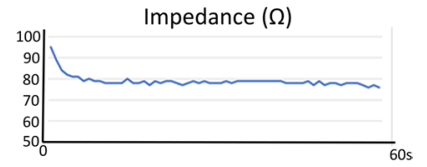

Fig. 1 The DiamondTemp catheter. The DiamondTemp ablation (DTA) (Medtronic, Inc., Minneapolis, Minnesota) catheter tip consists of a $4.1 \mathrm{~mm}$ distal electrode comprised of platinum:iridium (Pt:Ir), chemical vapour deposit (CVD) diamond network, 6 external thermocouples (TCs) and 6 saline irrigation ports. The CVD network allows thermal energy transfer that is $200-400$ times faster than achieved with other catheter systems. The catheter provides temperature control to maintain $60{ }^{\circ} \mathrm{C}$ at the tissue surface with real-time power modulation, as shown in the representative graphs of power, temperature and impedance change over the course of a single $60 \mathrm{~s}$ ablation lesion 
allowed it [9]. In brief, the Ensite Precision Cardiac Mapping System (Abbott Medical, Inc., Minneapolis, MN) was used to construct maps with the Advisor HD Grid Mapping catheter (Abbott Medical, Inc., Minneapolis, MN). The DTA catheter (Medtronic, Inc., Minneapolis, MN) was used for ablation. The DTA generator is a temperature-controlled closed-loop system that modulates power to reach and sustain a programmable tip-tissue temperature (default at $60{ }^{\circ} \mathrm{C}$ ) and an irrigation pump that operates at $8 \mathrm{ml} / \mathrm{min}$ while RF energy is delivered. The default DTA parameters were to achieve a target temperature of $60^{\circ} \mathrm{C}$ for a maximum of $60 \mathrm{~s}$ with initial power ramped to $50 \mathrm{~W}$ per lesion. The ablation duration was operator dependent with termination if bipolar electrogram (EGM) reduction or excessive impedance reduction of more than $20 \Omega$ was observed. Metrics recorded for each ablation lesion included power, impedance and temperature from all 6 thermocouples collected at a rate of $1 \mathrm{~Hz}$.

Substrate map data collected for each case included preand post-ablation bipolar and unipolar total scar area (TSA), borderzone area (BZA), dense scar area (DSA), late potential area (LPA) and mean bipolar and unipolar voltages of the ablation target area (ATA). Regular bipolar voltage definitions were used to describe preserved myocardial voltage $(>1.5 \mathrm{mV})$ and dense scar $(<0.5 \mathrm{mV})$ with LPs identified as isolated component $\geq 20 \mathrm{~ms}$ after the end of surface QRS with a detection sensitivity of $0.15 \mathrm{mV}$ [10]. Substrate map areas were measured offline post-procedure using the Ensite Precision Cardiac Mapping System (Abbott Medical, Inc., Minneapolis, MN) surface planimetry tool.

We used programmed electric stimulation (PES) from the right ventricular apex and outflow tract with 2 different drive cycle lengths (600 and $400 \mathrm{~ms}$ ) and introduction of up to 3 extrastimuli until a ventricular effective refractory period or a coupling interval of $200 \mathrm{~ms}$ was reached, without the use of isoproterenol. Clinical VT was determined from the available 12-lead ECG or VT cycle length in the ICD memory. All other monomorphic VTs including polymorphic VT were deemed non-clinical. Non-clinical monomorphic and haemodynamically stable VTs inducible during the procedure were also targeted for ablation. Complete elimination of all clinical and non-clinical monomorphic VTs was defined as complete success. Elimination of the clinical VT only was defined as partial success. Re-induction of clinical VT despite ablation was defined as procedural failure. Major procedure-related complications were defined as those necessitating additional interventions and leading to prolonged hospitalisation.

Post-procedure, ICD programming was left unchanged to facilitate analysis of individual response to ablation, with follow-up for a minimum of 6 months post-ablation. Postprocedure all patients remained on the previously ineffective anti-arrhythmic drug therapy that was administered for at least 6 months prior to ablation. ICD data for the total number of device detected VT episodes, number of device treated VT episodes, VT tachycardia cycle length, number of ATP episodes and number of shocks were collected during the follow-up period and compared to the pre-ablation 6 month period.

\subsection{Statistical analysis}

Continuous variables are expressed as mean $\pm \mathrm{SD}$ or median \pm IQR if necessary. Statistical significance was assessed using the unpaired Student's t-test or Mann-Whitney test if necessary. Categorical variables, expressed as numbers or percentages, were analysed using the chi-square test, Fisher's exact test or McNemar test for paired comparison. Univariate analysis of variables was performed. Cumulative event rates were calculated according to the Kaplan-Meier method. Hazard ratios with corresponding 95\% confidence intervals (CIs) are presented. $p$ Value $<0.05$ defined statistical significance. Statistical analysis was performed using MedCalc and Statview 5.0 statistical software.

\section{Results}

\subsection{Patient and procedural characteristics}

In total, 10 patients (mean age 73.4 years (range 64-84)) were recruited over 3 months (Table 1). The mean LVEF was $28.6 \pm 8.4 \%, 4$ patients were classified as high risk and 6 as intermediate risk using the PAINESD score, with no patient requiring haemodynamic support for the procedure [11]. All patients were established on optimal AAD therapy. The procedural characteristics are summarised in Table 1. In all cases, clinical VT was inducible with an average of 2.1 conduction channels identified per case with the described mapping strategies [9].

\subsection{Ablation lesion data}

The DTA lesion characteristics are summarised in Table 2. As the DTA generator provides power, temperature and impedance data every second, there were $2450 \pm 1241$ data time points analysed per procedure. The average number of ablation lesions per procedure was $51.8 \pm 17.7$ with an average individual ablation duration of $44.5 \pm 11.1 \mathrm{~s}$. The average power and temperature as well as the maximum power and temperature reached per ablation lesion are shown. The time taken to reach the average maximum temperature per ablation of $55.2 \pm 2.0{ }^{\circ} \mathrm{C}$ was on average $25.8 \pm 8.0 \mathrm{~s}$. Similarly, the time taken to reach the average lowest impedance reduction per ablation of $11.2 \pm 2.4 \Omega$ was on average $23.0 \pm 7.0 \mathrm{~s}$. 
Table 1 Baseline and procedural characteristics

\begin{tabular}{|c|c|}
\hline Age at ablation, years & $73.4 \pm 6.3$ \\
\hline Female sex, $n(\%)$ & $1(10)$ \\
\hline \multicolumn{2}{|l|}{ Ethnic Background } \\
\hline Caucasian, $n$ & $7(70)$ \\
\hline South Asian, $n$ & $3(30)$ \\
\hline Creatinine, $\mu \mathrm{mol} / \mathrm{L}$ & $119.8 \pm 34.3$ \\
\hline \multicolumn{2}{|l|}{ Aetiology } \\
\hline Ischaemic cardiomyopathy, $n$ & $10(100)$ \\
\hline \multicolumn{2}{|l|}{ Past medical history } \\
\hline AF/flutter, $n$ & $4(40)$ \\
\hline Hypertension, $n$ & $4(40)$ \\
\hline Diabetes, $n$ & $3(30)$ \\
\hline COPD, $n$ & $1(10)$ \\
\hline Cerebrovascular disease, $n$ & $0(0)$ \\
\hline $\mathrm{ICD}, n$ & $4(40)$ \\
\hline CRT-D, $n$ & $6(60)$ \\
\hline Pre-ablation ATP, $n$ & $9(90)$ \\
\hline Pre-ablation shock, $n$ & $4(40)$ \\
\hline Mean LVEF, \% & $28.6 \pm 8.4$ \\
\hline \multicolumn{2}{|l|}{ NYHA classification } \\
\hline $\mathrm{I}, n$ & $5(50)$ \\
\hline II, $n$ & $2(20)$ \\
\hline III, $n$ & $3(30)$ \\
\hline $\mathrm{IV}, n$ & 0 \\
\hline Average number of clinical VTs & $1.4 \pm 0.5$ \\
\hline Mean VT cycle length, milliseconds & $337.1 \pm 73.5$ \\
\hline Amiodarone at enrolment, $n$ & $9(90)$ \\
\hline B-blocker at enrolment, $n$ & $10(100)$ \\
\hline \multicolumn{2}{|l|}{ PAINESD Score ${ }^{13}$} \\
\hline Low risk $(\leq 8)$ & 0 \\
\hline Intermediate risk $(9-14)$ & 6 \\
\hline High risk $(\geq 15)$ & 4 \\
\hline$*$ Average procedure time, $\min$ & $179.4 \pm 52.3$ \\
\hline Average fluoroscopy time, min & $24.9 \pm 8.5$ \\
\hline Average substrate map points collected, $n$ & $26,165 \pm 11,384$ \\
\hline Average substrate map points used, $n$ & $2344 \pm 917$ \\
\hline Clinical VT induced, $n$ & $10(100)$ \\
\hline Conduction channels identified, $n$ & $2.1 \pm 1.2$ \\
\hline Late potentials identified, $n$ & $10(100)$ \\
\hline Decrementing evoked potentials mapped, $n$ & $9(90)$ \\
\hline Successful activation map, $n$ & $10(100)$ \\
\hline Successful pace map, $n$ & $9(90)$ \\
\hline Successful entrainment map, $n$ & $3(30)$ \\
\hline
\end{tabular}

*Defined as time from vascular access to catheter removal. Values are mean $\pm \mathrm{SD}$ or $n(\%)$

\subsection{Substrate map analysis}

Substrate map data analysis is summarised in Table 3. There was a significant reduction in the average bipolar BZA of $4.4 \mathrm{~cm}^{2} / 12 \%(p=0.026)$ and bipolar LPA of $3.5 \mathrm{~cm}^{2} / 83 \%$ $(p=0.0449)$ post-ablation. Figure $2 \mathrm{a}$ shows representative substrate and LP maps pre- and post-ablation. There
Table 2 Ablation lesion data

\begin{tabular}{ll}
\hline Average number of ablations/procedure, $n$ & $51.8 \pm 17.7$ \\
Average total ablation time, min & $40.8 \pm 20.5$ \\
Individual ablation duration, $\mathrm{s}$ & $44.5 \pm 11.1$ \\
Average ablation data points, $n$ & $2450 \pm 1241$ \\
Average power delivered, Watts & $47.8 \pm 1.2$ \\
Maximum power delivered, Watts & $51.8 \pm 1.3$ \\
Average temperature, ${ }^{\circ} \mathrm{C}$ & $49.8 \pm 2.0$ \\
Maximum temperature reached, ${ }^{\circ} \mathrm{C}$ & $55.2 \pm 2.0$ \\
Time to maximum temperature, $\mathrm{s}$ & $25.8 \pm 8.0$ \\
Average number of ablations with tempera- & $25.7 \pm 8.2(52 \pm 14)$ \\
ture $>55^{\circ} \mathrm{C}, n$ (\%) & \\
Average ablation start impedance, $\Omega$ & $97.9 \pm 19.3$ \\
Average ablation lowest impedance, $\Omega$ & $86.6 \pm 18.4$ \\
Average ablation drop in impedance, $\Omega$ & $11.2 \pm 2.4$ \\
Time to lowest impedance, $\mathrm{s}$ & $23.0 \pm 7.0$ \\
Maximum temperature sensing, $\%$ & \\
TCL1 & $22.0 \pm 14.1$ \\
TCL2 & $25.2 \pm 19.3$ \\
TCL3 & $16.8 \pm 11.1$ \\
TCL4 & $9.6 \pm 4.9$ \\
TCL5 & $16.7 \pm 11.1$ \\
TCL6 & $9.7 \pm 6.6$ \\
Average irrigation fluid infusion, $\mathrm{ml}$ & $435.3 \pm 215.3$ \\
\hline
\end{tabular}

Values are mean \pm SD or n (\%). TCL thermocouple. TCL 1-3=distal; TCL 4-6=shaft

was a significant reduction in the mean bipolar $(0.14 \mathrm{mV}$ $(p=0.0007))$ and unipolar $(0.59 \mathrm{mV}(p=0.0072))$ voltages of the ATA post-ablation with Fig. 2b highlighting the reduction in signal attenuation from the DTA catheter. Complete procedural success was achieved in 9 procedures. No post-ablation stimulation protocol was performed at the end of 1 procedure due to a steam pop occurring after $48 \mathrm{~min}$ of ablation resulting in pericardial tamponade requiring drainage.

This complication occurred in a 69-year-old male with ICM and an ejection fraction of 33\% with a CRT-D device. He had a background history of chronic obstructive airways disease and presented with 4 episodes of ATP and 1 shock for VT with a TCL of $352 \mathrm{~ms}$ with a high PAINESD score of 19. The substrate map confirmed inferior-septal scar and the VT activation map identified the VT isthmus extending from the septal borderzone to the inferior-lateral borderzone. In total, there were 59 ablation lesions in this case, with an average individual ablation lesion duration of $49 \mathrm{~s}$, with an average $11.8 \Omega$ impedance drop per lesion. The final ablation lesion resulted in a steam pop where the maximum temperature reached was $60{ }^{\circ} \mathrm{C}$ with an impedance reduction of 20 $\Omega$ targeting the VT isthmus. This was a $44 \mathrm{~s}$ duration lesion which achieved a $15 \Omega$ reduction within $10 \mathrm{~s}$ after initiation of index ablation, suggesting excessive catheter-tissue force contact. Pericardial tamponade was confirmed followed by 
Table 3 Substrate map data

\begin{tabular}{llcl}
\hline & Pre-ablation & Post-ablation & $p$ Value \\
\hline Average bipolar TSA, cm ${ }^{2}$ & $42.0 \pm 26.4$ & $41.2 \pm 24.2$ & $\mathrm{~ns}$ \\
Average bipolar DSA, cm ${ }^{2}$ & $31.4 \pm 23.4$ & $36.0 \pm 22.2$ & 0.0113 \\
Average bipolar BZA, cm ${ }^{2}$ & $10.7 \pm 5.8$ & $6.2 \pm 4.2$ & 0.0255 \\
$\%$ Bipolar BZA of TSA & $28 \pm 11$ & $16 \pm 11$ & 0.0260 \\
Average unipolar TSA, cm ${ }^{2}$ & $44.1 \pm 25.5$ & $42.0 \pm 20.8$ & $\mathrm{~ns}$ \\
Average unipolar DSA, cm ${ }^{2}$ & $39.7 \pm 25.3$ & $39.5 \pm 22.1$ & $\mathrm{~ns}$ \\
Average unipolar BZA, cm & 2 & $2.6 \pm 3.0$ & $\mathrm{~ns}$ \\
\% Unipolar BZA of TSA & $4.3 \pm 2.3$ & $9 \pm 9$ & $\mathrm{~ns}$ \\
Average bipolar LPA, cm & & $0.7 \pm 0.9$ & 0.0449 \\
Average bipolar voltage of ATA, mV & $13 \pm 10$ & $0.30 \pm 0.15$ & 0.0007 \\
Average unipolar voltage of ATA, mV & $4.2 \pm 4.9$ & $3.20 \pm 1.10$ & 0.0072 \\
\hline
\end{tabular}

Values are mean $\pm \mathrm{SD}$

(a)

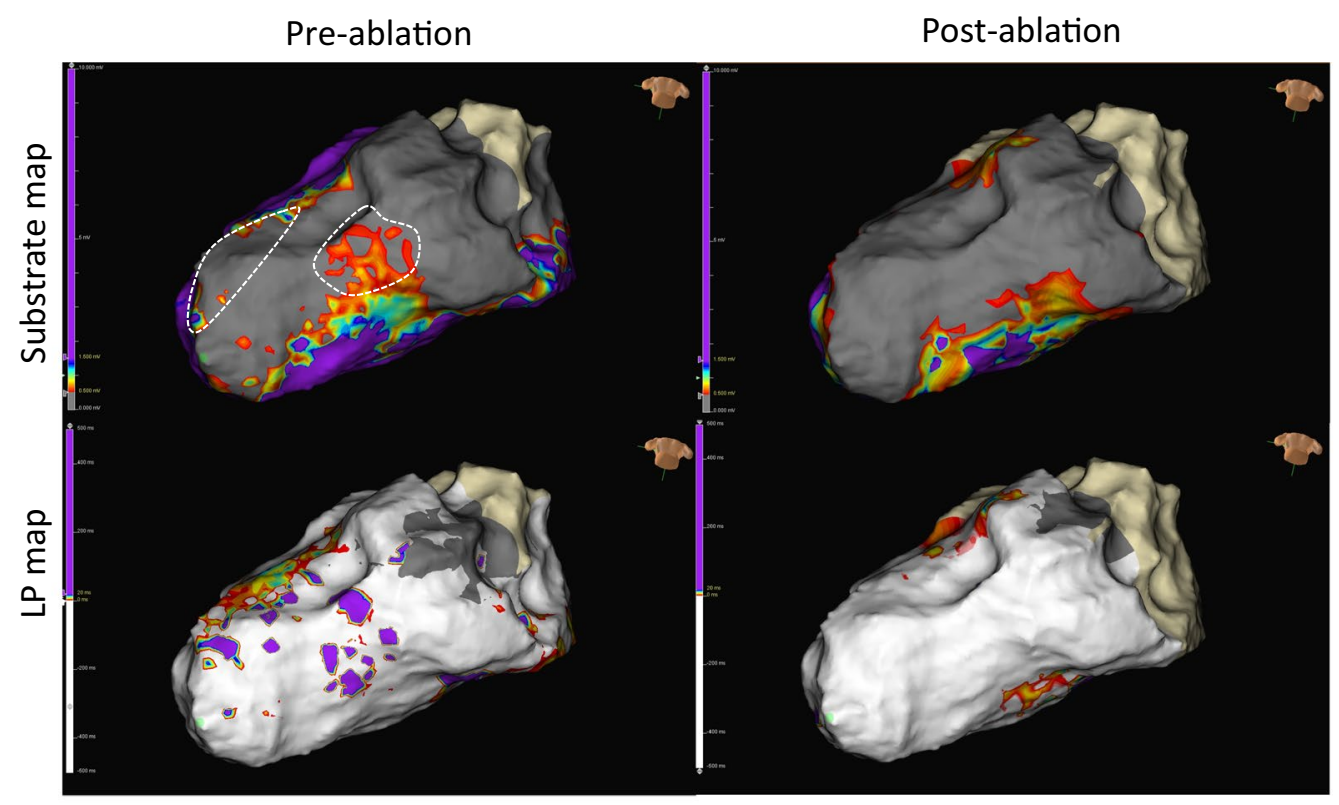

(b)
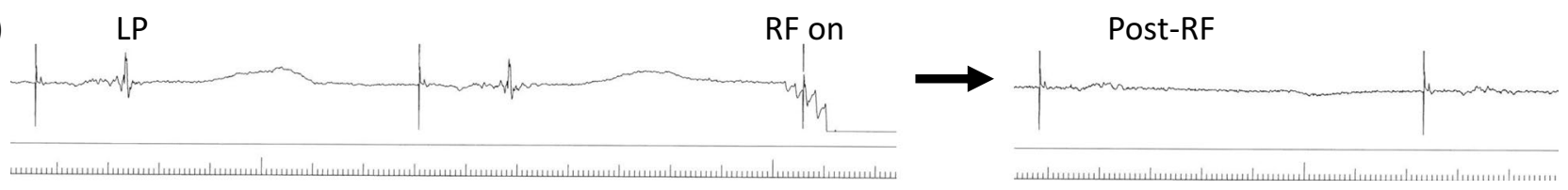

Fig. 2 Representative case. Substrate and LP maps are shown in a representative case pre- and post-ablation, using standard LV bipolar voltage criteria, with the ATA highlighted with the dashed white line. Post-ablation, the BZA has been effectively reduced from 7.5 to $3.2 \mathrm{~cm}^{2}$ with a corresponding increase in the DSA. The pre-ablation LPA $\left(5.8 \mathrm{~cm}^{2}\right)$ has been completely eliminated. The mean bipolar

intravenous protamine administration to reverse the unfractionated heparin as the pericardial drain was inserted. Approximately $100 \mathrm{ml}$ of blood was autotransfused and the patient stabilised. The drain was removed the following day and the patient recovered well. voltage of the ATA pre-ablation was $0.43 \mathrm{mV}$ and effectively reduced to $0.36 \mathrm{mV}$ post-ablation. b Electrogram amplitude attenuation of the bipolar LP signal from the DTA distal high-resolution electrode is shown $(\mathrm{LP}=$ late potential; $\mathrm{ATA}=$ ablation target area; $\mathrm{BZA}=$ borderzone area; DSA = dense scar area)

\subsection{Follow-up data}

Figure 3 highlights the individual reductions in total device detected VT and device treated VT episodes. All patients underwent a minimum of 6 months follow-up with a mean of 
(a)

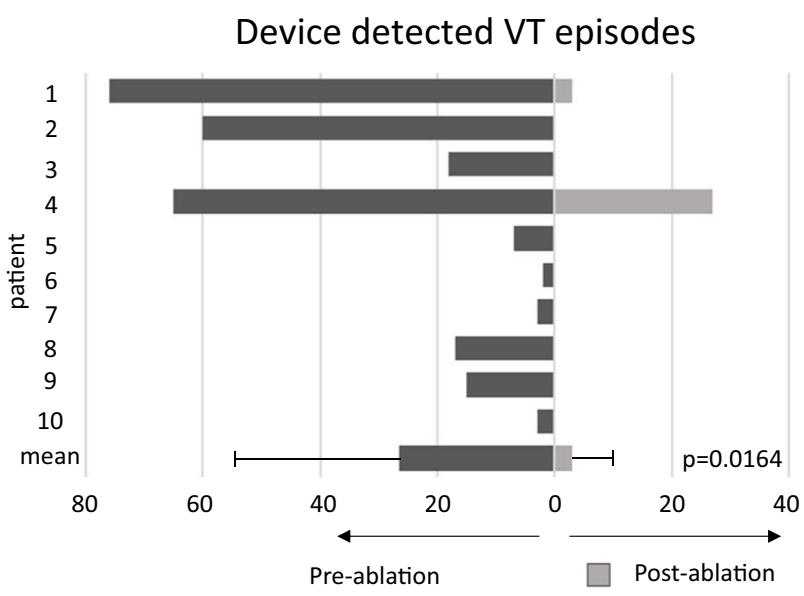

Fig. 3 Follow-up data. Histograms showing individual a device detected VT episodes and $\mathbf{b}$ device treated VT episodes within the 6 months pre-ablation period compared to the minimum 6 months post-ablation follow-up. Pre-ablation device detected VT episodes $n=266$; median 16 [IQR 3-57]. Post-ablation device detected VT

$214 \pm 33$ days. In the 6 months prior to ablation, there were a total of 266 device detected VT episodes, with a mean VT cycle length of $337 \pm 71 \mathrm{~ms}$, of which 128 were device treated VT episodes (120 ATP; 8 shocks). At final followup, a total of 33 device detected VT episodes were observed with a mean VT cycle length of $410 \pm 10 \mathrm{~ms}$, of which 30 were device treated VT episodes (27 ATP; 3 shocks). A significant $88 \%$ reduction in total VT episodes $(n=266$ median 16 [IQR 3-57] to $n=33$ median $0 ; p=0.0164$ ) and $77 \%$ reduction in ICD therapies $(n=128$ median 5 [IQR 2-15] to $n=30$ median $0 ; p=0.0181$ ) were observed. No deaths occurred in the study cohort.

\section{Discussion}

This single-centre study reports the first in-human experience of temperature-guided irrigated RF ablation for VT ablation using the DTA system. The novel design of the DTA catheter with an array of externalised thermocouples situated directly at the tip-tissue interface permits reintroduction of temperature-controlled irrigated RF ablation. The main findings of this study are that temperature-controlled irrigated RF ablation (1) delivers adequate power titration to achieve and maintain target tissue temperature and resultant tissue impedance reductions, (2) is effective in eliminating the VT substrate, and (3) results in a significant reduction in intermediate-term VT burden and ICD therapies.

Effective ablation lesions are produced when irreversible tissue damage occurs; at tissue temperatures of $>50^{\circ} \mathrm{C}$ [12]. The DTA system operates in a 'temperature control mode', (b)

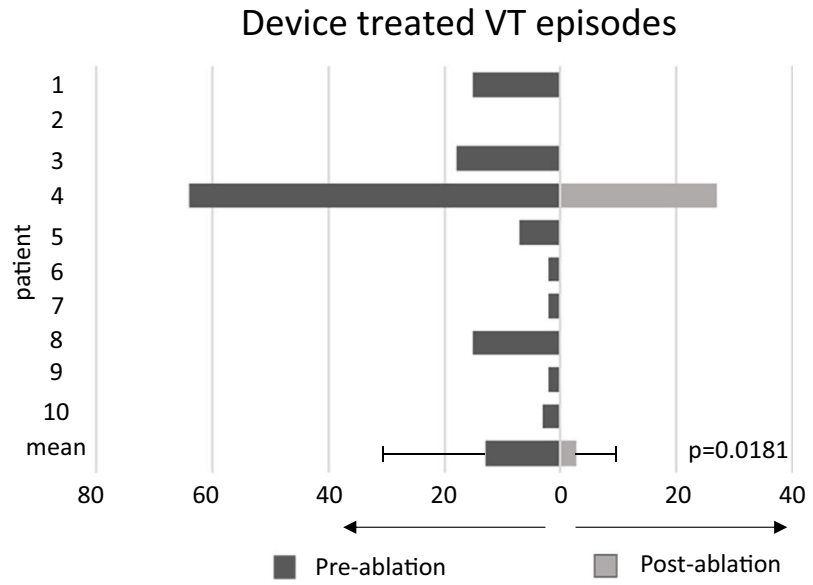

episodes $n=33$; median $0(p=0.0164)$. Pre-ablation device treated VT episodes $n=128$ median 5 [IQR 2-15]. Post-ablation device treated VT episodes $n=30$; median $0 \quad(p=0.0181)$. Values are mean $\pm \mathrm{SD}$.

with a constant feedback loop between the catheter thermocouples and the generator that optimises power output every $20 \mathrm{~ms}$. This ensures that effective lesions are created without excessive catheter tip temperatures. We were able to achieve an average temperature of $49.8^{\circ} \mathrm{C}$ with an average maximum temperature of $55.2{ }^{\circ} \mathrm{C}$ per ablation lesion. The time taken to achieve this maximum temperature was on average 25.8 s post-RF delivery. Interestingly, we observed the lowest impedance reductions at a similar time point of $23 \mathrm{~s}$.

The efficacy and safety of the DTA system has been reported for atrial fibrillation ablation [5, 7]. The DIAMOND-AF study recently reported the noninferiority of the DTA system to a force-sensing ablation system for pulmonary vein isolation [5]. The ablation recommendations in DIAMOND-AF were to achieve a target temperature of $60{ }^{\circ} \mathrm{C}$, and the average individual ablation duration was $14.7 \pm 5.3 \mathrm{~s}$. Based on preclinical data from computational modelling, thermochromic gel and porcine thigh preparation experiments, we proposed $60 \mathrm{~s}$ lesions that were guided by bipolar EGM and impedance changes [8].

\subsection{Independent relationship between Impedance and temperature change in lesion formation}

Impedance reduction is routinely monitored in clinical practice to assess RF ablation delivery having been shown to be an important indicator of successful lesion formation [13-15]. Previous studies have demonstrated the correlation between impedance reduction and lesion dimension with impedance reductions of $10 \Omega$ regarded as a marker of adequate lesion formation $[14,15]$. The initial impedance 
reduction during $\mathrm{RF}$ ablation is larger when greater catheter contact is achieved [14]. Indeed, it has been reported that impedance reductions of $\geq 10 \Omega$ cannot be achieved with contact forces less than $5 \mathrm{~g}$ under any power setting [16]. Preclinical data using the DTA system in a porcine thigh preparation shows a linear relationship between impedance reduction, duration of RF delivery and lesion depth [8]. Therefore, despite the non-contact force nature of the DTA catheter, adequate impedance reductions would support effective tissue contact.

Through design, the DTA catheter is expected to provide rapid thermal diffusivity and temperature feedback to allow for high power energy delivery. We observed a single steam pop that resulted in pericardial tamponade suggesting insufficient thermal feedback and power titration. Figure 4 represents a scatter plot of all 518 ablation lesions delivered within this study and $322(62 \%)$ lesions resulted in impedance reductions greater than $10 \Omega$. As termination of RF delivery was operator guided and not an automated process, 18 (3\%) lesions resulted in impedance reductions greater than $20 \Omega$. Interestingly, we observed a poor correlation between the maximum temperature reached and the impedance reduction per ablation lesion. This observation suggests that the catheter tip-tissue interface temperature alone may not be a solely reliable marker for safe and effective DTA lesion delivery. Indeed, the increased thickness of ventricular tissue, compared to atrial tissue, may facilitate increased temperatures farther from the catheter tip that cannot be detected by the externalised thermocouples.

Computational modelling studies applying the DTA system have shown that tip-tissue interface temperatures ranging from 52.7 to $60.3{ }^{\circ} \mathrm{C}$ will result in maximum internal tissue temperatures ranging from 82.8 to $94.0^{\circ} \mathrm{C}$ [8]. Furthermore, applying DTA lesions with a target temperature of $60{ }^{\circ} \mathrm{C}$ and maximum power of $50 \mathrm{~W}$ to a thermochromic gel preparation achieved tip-tissue interface temperatures of up to $64{ }^{\circ} \mathrm{C}$ with no application producing internal tissue temperatures greater than $80{ }^{\circ} \mathrm{C}$ [8]. These lesion applications were limited to $15 \mathrm{~s}$, and the longer application duration delivered with lesion $\mathrm{X}$ may have resulted in excessive tissue temperature despite an optimal tip-tissue interface temperature. The mechanism for this may have been excessive resistive heating which manifested as an excessive reduction in impedance.

We therefore propose that with temperature-guided irrigated RF ablation for VT ablation, both maximum temperature and impedance changes, are utilised to tailor the ablation duration. Our ablation protocol was to deliver $60 \mathrm{~s}$ ventricular lesions that were guided by bipolar EGM and impedance changes, and based on these parameters, the average individual ablation duration was $44.5 \pm 11.1 \mathrm{~s}$. This ablation duration is significantly longer than the $14.7 \pm 5.3 \mathrm{~s}$ average individual ablation duration reported for atrial ablation in the DIAMOND-AF study where ablation termination guidance was 3 to $5 \mathrm{~s}$ beyond electrogram amplitude attenuation of 75 to $80 \%$ [5]. Based on the current commercially available DTA generator and the data that is provided in real-time, our data study would support individual ventricular ablation durations limited to impedance reductions of no greater than $15 \Omega$, as was the case in $84 \%$ of lesions in this study.

\subsection{Importance of direct temperature sensing from catheter tip thermocouples}

The importance of direct temperature sensing from both the shaft thermocouples and distal tip thermocouples is reflected in our data. Of the total maximum temperatures recorded for all lesions, only $64 \%$ were from the 3 distal tip thermocouples, while the remainder were detected on the
Fig. 4 Temperature versus Impedance. Scatter plot of all 518 ablation lesions demonstrating a poor correlation between maximum temperature and impedance decrease ( $\mathrm{r}$ coefficient $=0.36 ; p$ value $=0.01$ ). Lesion $\mathrm{X}$ identifies ablation lesion resulting in steam pop and pericardial tamponade

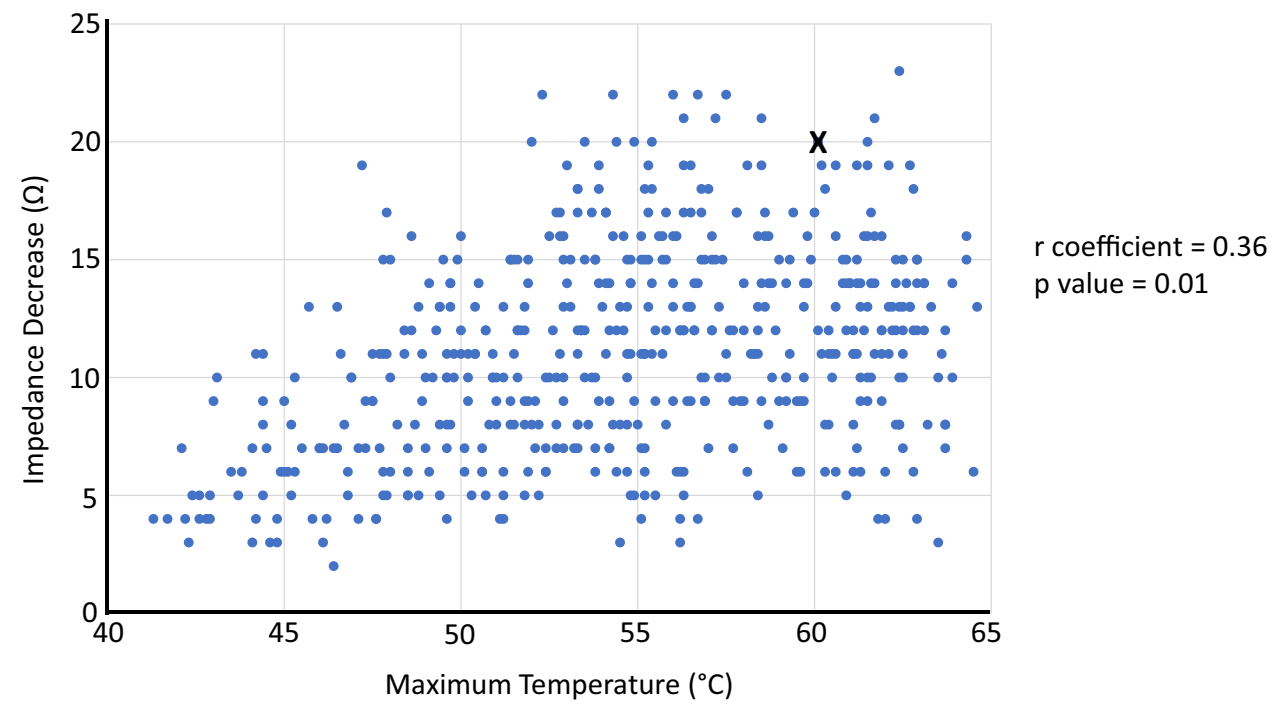


externalised shaft thermocouples. We observed a poor correlation between catheter orientation to tissue in predicting which thermocouple would detect the highest temperature. In case 8 , the target for ablation was the apical and septal mid-LV and $88 \%$ of the maximum temperatures detected were on the distal thermocouples. In case 5, where the septal and apical LV was targeted with a similar catheter orientation perpendicular to the myocardium, only $68 \%$ of the maximum temperatures detected were on the distal thermocouples. However, in case 1 where the ablation target area was the inferior mid-LV with a similar catheter orientation perpendicular to the tissue, only $54 \%$ of the maximum temperatures detected were on the distal thermocouples. On the contrary in cases 2 and 10, where the catheter tip was parallel to the myocardium with the ablation target area at the lateral basal LV and inferoseptal basal LV, 56\% and 68\% of the maximum temperatures detected were on the distal thermocouples, respectively.

A comparison of the time required to reach maximum temperature in cases $(n=6)$ of a good correlation of catheter orientation to the contacting/sensing thermocouples (301 individual ablation lesions) versus cases $(n=4)$ with a poor correlation of catheter orientation to the contacting/ sensing thermocouples (217 individual ablation lesions) was performed. The time taken to reach maximum temperature was significantly longer in the cases of poor catheter orientation correlation to the contacting/sensing thermocouples $(30.2 \pm 19.1 \mathrm{~s}$ vs $24.8 \pm 17.7 \mathrm{~s} ; p=0.001)$ suggesting the poor correlation may be due to reduced or angulated catheter contact or the tip was embedded within trabeculated ventricular structures. However, this conclusion is speculative due to the absence of real-time intracardiac imaging, contact force or force vector data to identify which thermocouple(s) had the real, tightest catheter-tissue contact. These observations do however highlight the importance of the externalised shaft thermocouples in contributing to power modulation.

\subsection{Safety}

All patients included in this study were classified as intermediate or high risk using the PAINESD score with significant co-morbidities [11]. Apart from one pericardial tamponade which required a pericardial drain for $24 \mathrm{~h}$, there were no other steam pops or significant post-procedural complications. The DTA system delivers high power RF energy; however, because of the catheter tip CVD diamond network and resulting rapid heat dissolution, the irrigation flow rate remains low at $8 \mathrm{ml} / \mathrm{min}$. The average ablation fluid irrigation per case was $435.3 \mathrm{ml}$ which would represent a 3- to fourfold reduction compared to standard irrigated RF catheters such as the TactiCath (Abbott Medical, Inc., Minneapolis, MN) or Smart Touch (Biosense Webster, Inc.) ablation catheters [9]. Whether this reduction in fluid volume would translate into reduced post-procedural decompensated heart failure is yet to be shown.

\subsection{Efficacy}

The efficacy of the DTA lesions was reflected in the substrate and LP map analyses pre- and post- ablation along with the post-procedural VT inducibility testing. We demonstrated a $12 \%$ reduction in average bipolar BZA with a corresponding $15 \%$ increase in bipolar DSA post-ablation. Furthermore, the LPA was significantly reduced by $83 \%$. High density maps were generated using the Advisor HD Grid Mapping catheter, which has been associated with improved long-term post-procedural outcomes [7, 9]. Accordingly, we demonstrated a significant reduction in the mean bipolar and unipolar voltages of the ATA consistent with effective substrate modification. These findings were further supported by noninducibility of VT during end-point testing. The effectiveness in substrate elimination and VT non-inducibility postprocedure translated into positive clinical outcomes over the follow-up period of 6-10 months with a significant reduction in total VT episodes and ICD therapies.

\subsection{Study limitations}

This study was a feasibility study designed to explore the ability of the DTA system to deliver effective temperaturecontrolled irrigated RF ablation for VT ablation. As such, the current study has a small sample size, included patients with ICM only, and follow-up was limited to a minimum of 6 months per patient. Furthermore, post-procedure ICD programming was left unchanged to facilitate analysis of individual responses to ablation; however, this may have resulted in the relatively common post-ablation observation of underdetected slower VTs. The DTA system was not compared to other ablation catheters that can also deliver high power and additional contact force data. Larger comparative studies with formal hypothesis and statistical power calculations are required to definitively investigate the safety and efficacy of the DTA system. Future randomised controlled trials are required to compare the performance of the DTA system against conventional RF ablation catheters. Studies are also required to assess the effectiveness and safety of the DTA system in patients with a non-ischaemic VT substrate.

\section{Conclusions}

This is the first-in-human study to report the ablation characteristics, efficacy in substrate elimination and intermediateterm success rates of temperature-controlled irrigated RF for VT ablation. Larger trials are ongoing to confirm safety and long-term effectiveness of this novel ablation technology. 
The DTA system delivered power-modulated, temperaturecontrolled ablation lesions with associated impedance reductions that resulted in successful substrate elimination and complete elimination of all clinical and non-clinical VTs. These findings were substantiated by the intermediate follow-up outcomes with a reduction in overall VT burden and ICD therapies.

\section{Declarations}

Conflict of interest The authors declare no competing interests.

Open Access This article is licensed under a Creative Commons Attribution 4.0 International License, which permits use, sharing, adaptation, distribution and reproduction in any medium or format, as long as you give appropriate credit to the original author(s) and the source, provide a link to the Creative Commons licence, and indicate if changes were made. The images or other third party material in this article are included in the article's Creative Commons licence, unless indicated otherwise in a credit line to the material. If material is not included in the article's Creative Commons licence and your intended use is not permitted by statutory regulation or exceeds the permitted use, you will need to obtain permission directly from the copyright holder. To view a copy of this licence, visit http://creativecommons.org/licenses/by/4.0/.

\section{References}

1. Sapp JL, Wells GA, Parkash R, et al. Ventricular tachycardia ablation versus escalation of antiarrhythmic drugs. N Engl J Med. 2016;375:111-21.

2. Kuck KH, Schaumann A, Eckardt L, et al. Catheter ablation of stable ventricular tachycardia before defibrillator implantation in patients with coronary heart disease (VTACH): a multicentre randomised controlled trial. Lancet. 2010;375:31-40.

3. Reddy VY, Reynolds MR, Neuzil P, et al. Prophylactic catheter ablation for the prevention of defibrillator therapy. N Engl J Med. 2007;357:2657-65.

4. Haines DE, Verow AF. Observations on electrode-tissue interface temperature and effect on electrical impedance during radiofrequency ablation of ventricular myocardium. Circulation. 1990;82:1034-8.

5. Kautzner J, Albenque JP, Natale A, et al. A novel temperaturecontrolled radiofrequency catheter ablation system used to treat patients with paroxysmal atrial fibrillation. JACC Clin Electrophysiol. 2021;7:352-63.
6. Yokoyama K, Nakagawa H, Wittkampf FH, Pitha JV, Lazzara R, Jackman WM. Comparison of electrode cooling between internal and open irrigation in radiofrequency ablation lesion depth and incidence of thrombus and steam pop. Circulation. 2006;113:11-9.

7. Iwasawa J, Koruth JS, Petru J, et al. Temperature-controlled radiofrequency ablation for pulmonary vein isolation in patients with atrial fibrillation. J Am Coll Cardiol. 2017;70:542-53.

8. Verma A, Schmidt MM, Lalonde JP, Ramirez DA, Getman MK. Assessing the relationship of applied force and ablation duration on lesion size using a diamond tip catheter ablation system. Circ Arrhythm Electrophysiol 2021;14:e009541.

9. Proietti R, Dowd R, Gee LV et al. Impact of a high-density grid catheter on long-term outcomes for structural heart disease ventricular tachycardia ablation. J Interv Card Electrophysiol 2021.

10. Marchlinski FE, Callans DJ, Gottlieb CD, Zado E. Linear ablation lesions for control of unmappable ventricular tachycardia in patients with ischemic and nonischemic cardiomyopathy. Circulation. 2000;101:1288-96.

11. Muser D, Castro SA, Liang JJ, Santangeli P. Identifying risk and management of acute haemodynamic decompensation during catheter ablation of ventricular tachycardia. Arrhythm Electrophysiol Rev. 2018;7:282-7.

12. Leshem E, Zilberman I, Tschabrunn CM, et al. High-power and short-duration ablation for pulmonary vein isolation: biophysical characterization. JACC Clin Electrophysiol. 2018;4:467-79.

13. Bourke T, Buch E, Mathuria N, et al. Biophysical parameters during radiofrequency catheter ablation of scar-mediated ventricular tachycardia: epicardial and endocardial applications via manual and magnetic navigation. J Cardiovasc Electrophysiol. 2014;25:1165-73.

14. Reichlin T, Knecht S, Lane C, et al. Initial impedance decrease as an indicator of good catheter contact: insights from radiofrequency ablation with force sensing catheters. Heart Rhythm. 2014;11:194-201.

15. Yazaki K, Ejima K, Kanai M, et al. Impedance drop predicts acute electrical reconnection of the pulmonary vein-left atrium after pulmonary vein isolation using short-duration high-power exposure. J Interv Card Electrophysiol. 2020;59:575-84.

16. Shi LB, Wang YC, Chu SY et al. The impacts of contact force, power and application time on ablation effect indicated by serial measurements of impedance drop in both conventional and highpower short-duration ablation settings of atrial fibrillation. J Interv Card Electrophysiol 2021.

Publisher's note Springer Nature remains neutral with regard to jurisdictional claims in published maps and institutional affiliations. 